\title{
A Review on the Mechanical Behavior of Size-Dependent Beams and Plates using the Nonlocal Strain-Gradient Model
}

\author{
Tahereh Doroudgar Jorshari ${ }^{1}$ and Mir Abbas Roudbari ${ }^{2, *}$ \\ ${ }^{1}$ Faculty of Mechanical Engineering, Islamic Azad University, Takestan, Qazvin, Iran \\ ${ }^{2}$ School of Engineering, RMIT University, PO Box 71, Bundoora, VIC, 3083, Australia
}

\section{Article Info:}

Keywords:

Nonlocal strain-gradient continuum

mechanics model,

Mechanical loadings,

Micro-/nano-beams/-plates.

\section{Timeline:}

Received: October 29, 2021

Accepted: November 30, 2021

Published: December 01, 2021

Citation: Jorshari TD, Roudbari MA. A Review on the Mechanical Behavior of Size-Dependent Beams and Plates using the Nonlocal Strain-Gradient Model. J Basic Appl Sci 2021; 17: 184193

DOI: https://doi.org/10.29169/1927-5129.2021.17.18

\begin{abstract}
:
Nowadays, the mechanical characteristics of micro-/nano-structures in the various types of engineering disciplines are considered as remarkable criteria which may restrict the performance of small-scale structures in the reality for a certain application. This paper deals with a comprehensive review pertinent to using the nonlocal strain-gradient continuum mechanics model of size-dependent micro/nano-beams/-plates. According to the non-classical features of materials, using size-dependent continuum mechanics theories is mandatory to investigate accurately the mechanical characteristics of the micro-/nano-structures. Recently, the number of researches related to the analysis of micro-/nano-structures with various geometry including beams as well as plates is considerable. In this regard, the mechanical behavior of these structures induced by different loadings such as vibration, wave propagation, and buckling behavior associated with the nonlocal strain-gradient continuum mechanics model is presented in this review work. Proposing the most valuable literature pertinent to the nonlocal strain-gradient continuum mechanics theory of micro-/nano-beams/plates is the main objective of this detailed survey.
\end{abstract}

*Corresponding Author

E-mail: mir.abbas.roudbari@rmit.edu.au, m.a.roudbari@gmail.com

(c) 2021 Jorshari and Roudbari; Licensee SET Publisher.

This is an open access article licensed under the terms of the Creative Commons Attribution Non-Commercial License (http://creativecommons.org/licenses/by-nc/3.0/) which permits unrestricted, non-commercial use, distribution and reproduction in any medium, provided the work is properly cited. 


\section{INTRODUCTION}

Micro-/nano-electromechanical systems (M/NEMS) have been growing in various engineering applications such as mechanical, structural, aerospace, biomedical, as well as electrical, in which their mechanical behavior investigation can be considered as a challenging problem in their modeling. Also, to design various micro-/nano-structures such as micro-/nano-beams as well as micro-/nano-plates, functional knowledge of their physical (mechanical, electrical, thermal, etc.) features is mandatory [1-3]. It is important to mention that after the discovery of novel manufacturing technologies pertinent to size-dependent components, the number of practical applications related to using micro/nano-structures in various disciplines of engineering problems was considerable [4-6]. Furthermore, there are so many examples that can be shown in the design of components of some smart devices or machines including spacecraft, submarines, medical devices, bio/nano-sensors, electrical circuits, and so on. Many approaches can be employed to model the size-dependent structures including molecular dynamics, density functional theory, and tight-binding molecular dynamic [7-9] (peculiar to atomistic simulation schemes), continuum mechanics models, or their combination. Besides, using classical and non-classical continuum mechanical models are easier to model micro-/nano-structures than the atomistic models.

Researchers employed various size-dependent methods such as the nonlocal elasticity of Eringen, the strain gradient theory, the modified couple stress model, the micromorphic theory, as well as the nonlocal-stress gradient (as a hybrid model) procedure [10-20] to introduce the differences between classical and non-classical models. Many researchers studied the mechanical behavior of micro-/nano-structures based on the non-classical continuum mechanics models induced by different types of loadings including vibration [21-32], wave propagation [33-43], and buckling phenomenon [44-54] associated with linear and nonlinear approaches related to the kinematic relations. According to their hypothesis as well as obtained results, various methods such as analytical, semi-analytical, as well as numerical schemes were proposed to investigate the static/dynamic characteristics of their models.

Recently, researchers decided to utilize nonlocal straingradient models of small-scale effects such as the nonlocal-stress gradient method. This method has the advantages of both the nonlocal and strain gradient models together. Some scientists proposed that employing the nonlocal elasticity and the strain gradient models can change the stiffness behavior of the micro/nano-structures drastically, in particular in some specific small-scale parameters. Therefore, using the nonlocal strain-gradient model size-dependent model gives a more realistic model at micro/nano-scale. In this regard, there are many advantages, drawbacks, and key applications pertinent to using the nonlocalstress gradient size-dependent methods [55-60]. For example, this hybrid model contains both nonlocal and material length scale parameters and predict the stiffness-hardening influences using the length scale parameter [61-62]. Also, a nonlocal strain-gradient model is more accurate for modeling and analysis of micro/nano-structures using both stiffness reduction and enhancement influences [61-62]. Of course, for certain boundary conditions, the results are not accurate and correct. Moreover, some key applications of this theory are wave dispersion analysis of FGM, biological tissues, energy harvesting, M/NEMS cantilever actuators [24, 61-62].

There are several interesting papers in the literature pertinent to the review of micro/nano-structures [63-67]. It is significant to mention that the investigation of the important outcomes pertinent to the influences of the nonlocal-stress gradient size-dependent methods on various micro/nano-structures is the main motivation of this study as well as the major difference between the current review and published papers.

\section{NONLOCAL STRAIN-GRADIENT CONTINUUM MECHANICS THEORY}

Several procedures can be used in modelling smallscale structures such as molecular dynamics, density functional theory, as well as tight-binding MD $[9,68]$, which are proposed as atomistic simulation models and continuum mechanics. Moreover, as it was mentioned, there are many size-dependent effects including strain gradient method, couple stress theory, and micromorphic model. Using the mentioned sizedependent effects can be relevant to the application of micro-/nano-structures in various engineering disciplines. Indeed, based on the softening or hardening behavior of material and proposed boundary conditions used in various small-scale structures such as Euler-Bernoulli, Rayleigh, Timoshenko, higher-order beam theories as well as plate models, nonlocal Eringen, couple stress, strain-gradient, and micromorphic theories can be employed. 
Based on the above-mentioned, using a suitable sizedependent model is significant to scrutinize the mechanical characteristics of micro-/nano-structures at micro-/nano-sized scales. The nonlocal strain-gradient method has been utilized to investigate the nonlocal and strain gradient models, simultaneously. Using the higher-order theory by Lim et al. [69], the internal energy density potential $U_{E D}\left(\varepsilon_{i j}, \varepsilon_{i j}^{\prime}, e_{0} a\right)$ within the domain $V$ can be given as follows:

$U_{E D}\left(\varepsilon_{i j}, \varepsilon_{i j}^{\prime}, e_{0} a\right)=\frac{1}{2} \varepsilon_{i j} C_{i j k l} \int_{V} \alpha_{0}\left(\left|x-x^{\prime}\right|, e_{0} a\right) \varepsilon_{k l}^{\prime} d V$

$+\frac{l^{2}}{2} \varepsilon_{i j, m} C_{i j k l} \int_{V} \alpha_{1}\left(\left|x-x^{\prime}\right|, e_{1} a\right) \varepsilon_{k l, m}^{\prime} d V$

in which $e_{0} a, e_{1} a, C_{i j k l}, l$ are, the small-scale parameters, elastic modulus tensor, and material length scale parameter, respectively. Moreover, $\alpha_{0}\left(\left|x-x^{\prime}\right|, e_{0} a\right)$ and $\alpha_{1}\left(\left|x-x^{\prime}\right|, e_{1} a\right)$ are the nonlocal attenuation kernel functions and is the volume of the model. Furthermore, $\varepsilon_{i j}$ and $\varepsilon_{i j}^{\prime}$ are the Cartesian terms of the strain tensors. The total stress tensor will be proposed as follows:

$\tau_{x x}=\sigma_{x x}-\frac{d \sigma_{x x}^{1}}{d x}$,

where $\sigma_{x x}$ and $\sigma_{x x}^{1}$ are the classical and higher-order stresses, respectively. Based on Lim's theory, the final constitutive equation of the nonlocal strain-gradient model can be proposed as [69-79]:

$\left(1-(e a)^{2} \nabla^{2}\right) \tau_{x x}=E\left(1-(l)^{2} \nabla^{2}\right) \varepsilon_{x x}$.

in which $E, \tau_{x x}, \varepsilon_{x x}$ are, the elasticity modulus, the stress, and strain counterparts, respectively.

\subsection{Static Bending and Buckling}

In recent years, the investigation of linear and nonlinear bending, as well as buckling of micro/nano-structures based on the nonlocal strain-gradient method was carried out by some researchers. Many studies proposed analytical approaches to scrutinize their linear bending behavior. Lu et al. [76] studied the investigation of a nonlocal strain-gradient theory related to the micro-/nano-beams with consideration of the importance of higher-order terms using Euler-Bernoulli as well as Timoshenko beam models. Based on Hamilton's principle and the relevant boundary conditions, the relations were obtained. The effects of the nonlocal strain-gradient theory on the bending characteristics of the sandwich porous nanoplate using piezomagnetic face sheets as well as the first-order shear deformation model were proposed by Arefi et al. [77]. They used the power-law function to scrutinize changing the porosity along with the direction of thickness encapsulated by the Pasternak medium. Allam and Radwan [81] investigated the nonlocal strain-gradient model of bending as well as buckling of the FG curved micro-/nano-beam on an elastic foundation with viscoelastic effects and different boundary conditions. They employed the two powerlaw models to show any changes in material features associated with viscoelastic FG curved micro-/nanobeam. Xu et al. [82] proposed the bending and buckling characteristics of the micro-/nano-beams using the nonlocal strain-gradient elastic model. They proved that using the higher-order boundary conditions leads to having no significant effect on the bending deflection of the micro-/nano-beam.

Barretta et al. [83] examined the boundary conditions effects on nonlocal strain-gradient micro-/nano-beams. They indicated equivalence between a differential model as well as the nonlocal strain-gradient integral scheme using the proposed constitutive boundary conditions. Gao et al. [84] investigated nonlinear static bending characteristics of an FG porous micro-/nanobeams using physical fields and the nonlocal straingradient theory. They obtained the nonlinear relations using Hamilton's method. Beddia et al. [85] proposed a new hyperbolic in conjunction with two-unknown micro/nano-beam related to buckling and bending characteristics with consideration of the nonlocal straingradient theory. They utilized Navier's solution for the governing equations to solve them analytically based on the S-S boundary condition. Besides, several authors employed discretization methods to investigate the buckling as well as post-buckling characteristics of the micro-/nano-structures. Farajpour et al. [86] proposed the nonlocal strain-gradient theory for buckling behavior of an orthotropic micro-/nano-plate induced by the thermal environment. Based on the DQM procedure the equations were examined and solved. Bending/buckling/vibration characteristics of axially FG micro-/nano-beams with consideration of the nonlocal strain-gradient as well as Euler-Bernoulli beam theories were proposed by $\mathrm{Li}$ et al. [87]. According to the through-length grading model of the FG material, the mechanical behavior was investigated. Also, the buckling load, deflection, and frequency values were controlled with appropriate amounts of the power-law index. 
Moreover, numerical procedures were employed by researchers to scrutinize the nonlinear behavior of buckling, as well as post-buckling of micro-/nanostructures. Post-buckling, as well as bending behavior of nano-beams using nonlocal strain-gradient model based on the nonlinear influences, were carried out by Zhong et al. [88]. They scrutinized the multi-scale method of the Euler Bernoulli nano-beam based on the long deflection model. $\mathrm{Li}$ and $\mathrm{Hu}$ [89] studied the postbuckling features of FG nano-beams using nonlocal stress/strain gradient models with consideration of the nonlinear geometric associated with von Kármán theory. They used the physical neutral surface for removing the proposed coupling between bending/stretching associated with geometric nonlinearity related to the FG micro-/nano-structure. Mao and Zhang [90] investigated the buckling and post-buckling characteristics of FG graphene reinforced piezoelectric micro-/nano-plate using axial forces and the electric potential. They utilized the Halpin-Tsai parallel method to obtain the effective Young's modulus pertinent to each layer. They indicated that graphene platelet has an interesting influence on the buckling and post-buckling strength associated with the FG piezoelectric micro-/nano-plate.

\subsection{Vibration and Wave Propagation}

The following researches indicate the proposed works related to the linear and nonlinear free/forced vibration as well as wave propagation of the micro-/nanostructures.

Roudbari and Ansari [80] analyzed the physical characteristics of SWBNNT as a bio-/nano-sensor due to sensing attached micro/-nanoscale objects. Based on the various boundary conditions including SS-SS, $\mathrm{C}-\mathrm{C}$, and $\mathrm{C}-\mathrm{F}$, the vibrational characteristics of the model were examined. They used Rayleigh and Timoshenko beam models in their study. Furthermore, the nonlocal strain-gradient method was employed to show the size-dependent influences. Also, numerical methods were employed to investigate the mechanical properties of size-dependent structures. Figure 1a-c show the influences of the dimensionless amounts of the mass weight of attached nanoparticles on the relative frequency shift values. It was obvious that relative frequency shift values increase with an increase in the amounts of mass weight of attached nanoparticles, which was correct for nonlocal straingradient RBT and TBT. Moreover, the results of nonlocal strain-gradient TBT track the outputs of nonlocal strain-gradient RBT for all magnitudes of mass weight of the attached nanoparticle. Also, the discrepancy between the obtained relative frequency shift values for nonlocal strain-gradient RBT and TBT was observable in higher values of the mass weight of the attached nanoparticle. Furthermore, the C-C boundary condition had higher values than other case studies.

They also obtained the predicted first four dimensionless flexural frequencies of zigzag $(7,0)$ form SWBNNT as a bio-/nano-mass sensor which is provided in Table 1. Their obtained results were pertinent to S-S, bridged and cantilever types of boundary conditions and also nonlocal strain-gradient RBT and TBT models. It was clear that the C-F boundary condition case study had lower values of dimensionless flexural frequencies than other types of boundary conditions.
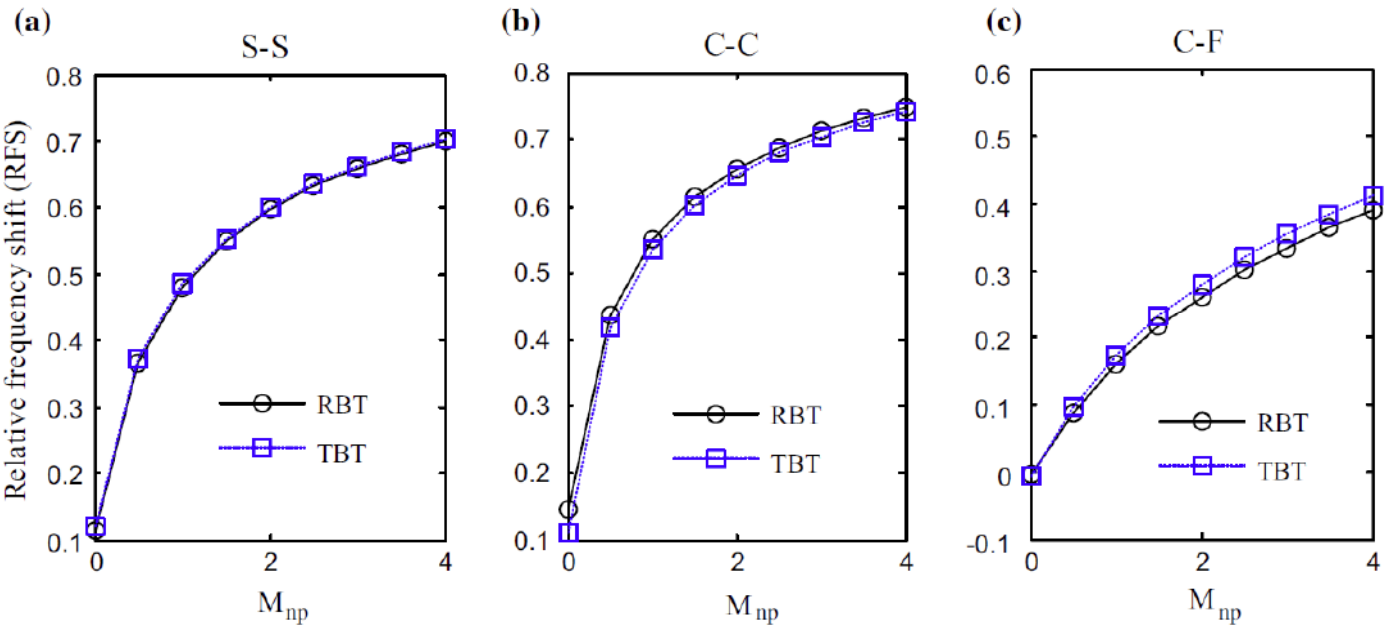

Figure 1: (a-c). The influences of the dimensionless amounts of the mass weight of attached nanoparticles on the relative frequency shift values. 
Table 1: Predicted First Four Dimensionless Flexural Frequencies of $(7,0)$ SWBNNT

\begin{tabular}{|c|c|c|c|c|c|}
\hline Mnp & Predicted results & $\sqrt{\bar{\omega}_{1}}$ & $\sqrt{\bar{\omega}_{2}}$ & $\sqrt{\bar{\omega}_{3}}$ & $\sqrt{\bar{\omega}_{4}}$ \\
\hline \multirow[t]{4}{*}{0} & NSRBT (S-S) & 3.0638 & 7.9321 & 11.1057 & 13.2447 \\
\hline & NSRBT (C-C) & 4.5834 & 9.0884 & 11.9654 & 13.8896 \\
\hline & NSTBT (C-C) & 4.478 & 8.3824 & 10.4365 & 12.902 \\
\hline & NSTBT (C-F) & 1.9122 & 4.4968 & 6.8242 & 9.6902 \\
\hline \multirow[t]{3}{*}{0.5} & NSRBT (S-S) & 2.6059 & 7.4691 & 10.8409 & 14.6669 \\
\hline & NSTBT (S-S) & 2.6144 & 7.1336 & 9.7565 & 12.8297 \\
\hline & NSRBT (C-C) & 3.792 & 8.7123 & 11.7311 & 13.7717 \\
\hline \multirow[t]{6}{*}{1} & NSRBT (S-S) & 2.3629 & 7.3305 & 10.772 & 14.6489 \\
\hline & NSTBT (S-S) & 2.3717 & 6.9872 & 9.6845 & 12.8075 \\
\hline & NSRBT (C-C) & 3.4059 & 8.6116 & 11.6785 & 13.7471 \\
\hline & NSTBT (C-C) & 3.3361 & 7.9014 & 10.1075 & 12.0748 \\
\hline & NSRBT (C-F) & 1.2484 & 3.9124 & 6.4756 & 10.2156 \\
\hline & NSTBT (C-F) & 1.2716 & 3.88 & 6.2655 & 10.2654 \\
\hline
\end{tabular}

Nonlinear vibration behavior of bi-directional FG micro/nano-beams with consideration of the nonlocal stress as well as micro-structural strain gradient effects was examined by Sahmani and Safaei [91]. They utilized GDQM as well as Galerkin procedures and also pseudo-arc-length continuation schemes to solve the proposed relations of motions. They studied the nonlocal strain-gradient of 2D FGM against different magnitudes with the nonlinear dynamic response of the axial material characteristics. Ghayesh et al. [92] investigated the coupled dynamics pertinent to nanofluid-conveying micro/nanotubes. Using the Beskok-Karniadakis model, the size-dependent influences of the nanofluid were proposed. Likewise, they used Coriolis acceleration effects based on the influences of the centrifugal acceleration. At a specific speed of nanofluid, the coupled bifurcation variation of the nanofluid-conveying micro/nanotubes was examined. Roodgar Saffari et al. [24] worked on the vibration behavior of fluid conveying SWCNT surrounded by a visco-Pasternak substrate with nonlinearity effects using the nonlocal strain-gradient model. They indicated that the size-dependent parameters have considerable effects on the dynamic behavior of the proposed model. The effect of variation of eigenvalue by velocity for different values of Winkler and damping parameters were illustrated in Figure 2. The real part (damping ratio) curve did not change at lower values of fluid speed but divergence at higher values of fluid speed. Also, by increasing damping parameters, total damping of the system increased, the imaginary part and the critical values of speed decreased. Also, the real part increased and divergence at lower values of fluid speed.

Barati et al. [61] worked on the forced vibration characteristics of the heterogeneous porous FG micro/nano-plates using the nonlocal stress-strain gradient theory. He examined the effects of the stiffnesssoftening/hardening behavior to scrutinize the mechanical features of the proposed model. Roudbari and Doroudgar Jorshari [93] analyzed the control behavior of SWCNT acted upon by a moving nanoparticle using the nonlocal strain-gradient as well as the Rayleigh beam theories with consideration of thermal and magnetic influences. They proved that the PZT patches as bio/nano-sensors can be connected to a charge amplifier for actuating the SWCNT which can be shown in Figure 3.

Wu et al. [94] proposed forced vibration behavior of FG graphene platelet-reinforced nanocomposite micro/nano-beams based on the nonlocal strain-gradient 

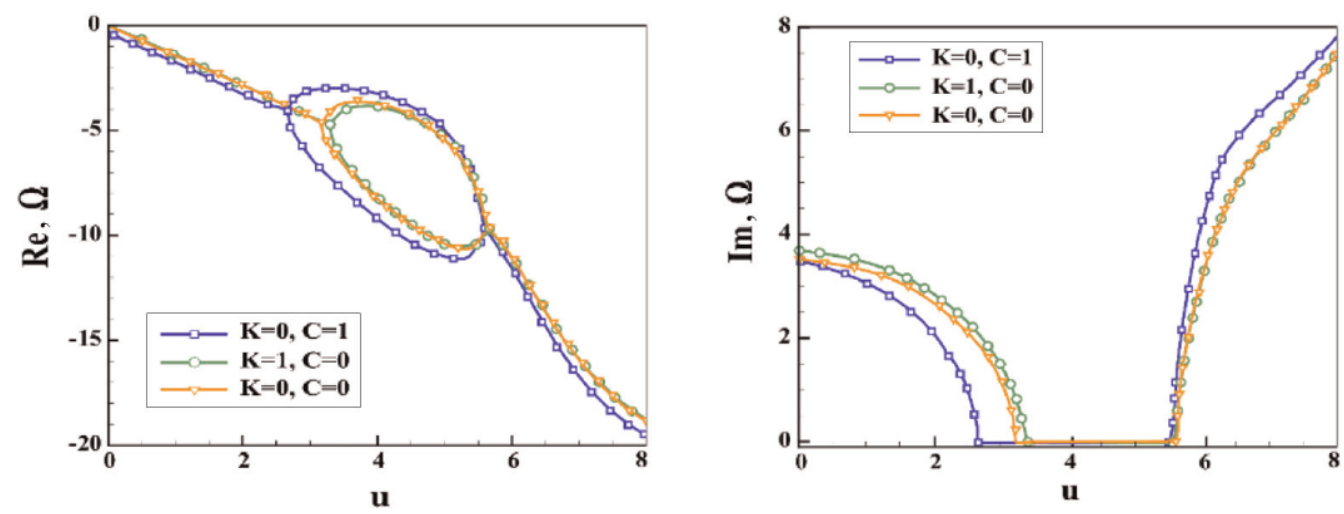

Figure 2: Effect of variation of eigenvalue by velocity for different value of Winkler and damping parameter.

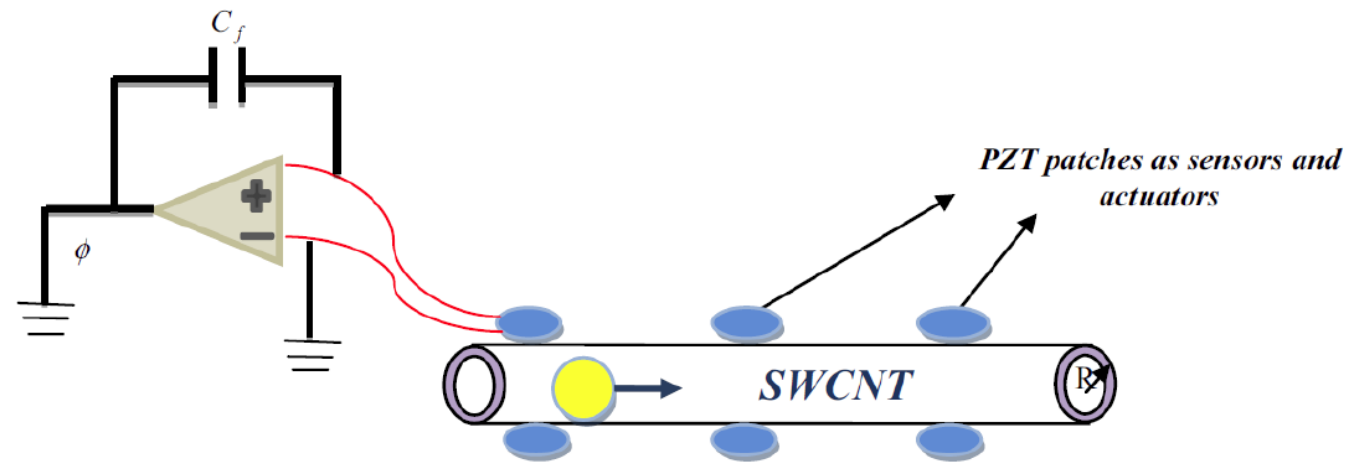

Figure 3: The PZT patches as sensors connected to charge amplifiers to actuate the SWCNT induced by a moving nanoparticle.

theory as well as the refined hyperbolic shear deformation beam procedure. The material features of laminated FG graphene platelet-reinforced nanocomposite micro-/nano-beams were obtained using the Halpin-Tsai method. Mahmoudpour [95] reported the nonlinear resonant behavior of a thick multi-layered nano-plate using the nonlocal straingradient model and the first-order shear deformation plate theory. The interactional vdW forces between the proposed adjacent layers of the nano-plate were considered. Vahidi-Moghaddam et al. [96] examined the nonlinear characteristics of forced vibration of the micro-/nano-beam using the nonlocal strain-gradient theory and the Euler-Bernoulli beam model for C-C boundary conditions. A reduced motion equation based on the central harmonic load as well as the Galerkin procedure was proposed.

Several works related to the analytical methods for the elastic wave propagation of the micro-/nano-structures have been carried out. Wave propagation behavior of the visco-elastic SWCNT using the nonlocal straingradient model was reported by Tang et al. [97]. They indicated that the blocking diameter value was related to the damping ratio, Winkler modulus, and the nonlocal strain-gradient scale parameters. The wave propagation characteristics of micro-/nano-beams for the Timoshenko beam model with consideration of the nonlocal strain-gradient theory were analyzed by Norouzzadeh et al. [98]. They showed that the classical beam model underestimates and overestimates the wave frequency values for without as well as with consideration of the nonlocal parameter, respectively.

On the other hand, discretization procedures were employed to show the mechanical characteristics of the elastic wave propagation responses with nonlinear effects of the micro-/nano-structures. Ebrahimi et al. [62] examined wave propagation of the FG nano-plates with consideration of the inhomogeneous effects and the nonlinear thermal influences based on the nonlocal strain-gradient model. Nonlinear modelling of the flexural wave propagation using the nonlocal straingradient scheme related to the Euler-Bernoulli and Timoshenko beam models was investigated by Huang et al. [99]. Also, Huang and Wei [100] worked on the flexural wave propagation modelling of the infinite micro-/nano-plate with consideration of the homogeneous effects and fractional nonlocal straingradient model. They employed the spatial and 
temporal fractional differential to indicate the spatial nonlocal and the history-dependent characteristics pertinent to the thermoelastic features of the micro/nano-structures.

\section{CONCLUSION}

A comprehensive survey of the nonlocal strain-gradient continuum mechanics model pertinent to the microInano-beams and -plates were examined. The proposed parts of this review are based on the nonlocal strain-gradient theory to show the size-dependent influences with consideration of different external loading including bending, buckling, vibration, and wave propagation. The recently published papers pertinent to using small-scale methods for micro-and nano-structures are considerable; thus, the current review is aimed to work on the proposed small-scale method employed in various beams and plates with different geometrical and physical parameters. Hope the present review help researchers to address the advantages, limitations, and deficiencies of using this procedure in the modelling of small-scale structures.

\section{REFERENCES}

[1] Hieu NM, Lam DV, Hien TT, Chinh ND, Quang ND, Hung NM, Phouc CV, Lee SM, Jeong JR, Kim C, Kim D. ZnTecoated $\mathrm{ZnO}$ nanorods: Hydrogen sulfide nano-sensor purely controlled by pn junction. Mater Des 2020; 191: 108628. https://doi.org/10.1016/j.matdes.2020.108628

[2] Zulhairun AK, Abdullah MS, Ismail AF, Goh PS. Chapter 1 Graphene and CNT Technology. Current Trends and Future Developments on (Bio-) Membranes Membrane Desalination Systems: the Next Generation 2019; 3-26. https://doi.org/10.1016/B978-0-12-813551-8.00001-2

[3] Ozaki JI. Chapter 2.4 - Catalytic Carbons - Cathode Catalytic Carbons. Handbook of Advanced Ceramics (Second Edition) Materials, Applications, Processing, and Properties 2013; 103-111.

https://doi.org/10.1016/B978-0-12-385469-8.00005-8

[4] Patra JK, Das G., Fraceto LF, Campos EVR, RodriguezTorres MDP, Acosta-Torres LS, Diaz-Torres LA, Grillo R, Swamy MK, Sharma S, Habtemariam S, Shin HS. Nano based drug delivery systems: recent developments and future prospects. J Nanobiotechnology 2018; 16: 71. https://doi.org/10.1186/s12951-018-0392-8

[5] Sheikhpour M., Arabi M., Kasaeian A., Rokn Rabei A., Taherian Z. Role of nanofluids in drug delivery and biomedical technology: methods and applications. Nanotechnol 2020; 13: 47-59. https://doi.org/10.2147/NSA.S260374

[6] Soto F, Wang J, Ahmed R, Demirci U. Medical micro/nanorobots in precision medicine. Adv Sci 2020. https://doi.org/10.1002/advs.202002203

[7] Chiu HY, Hung P, Postma Henk WC, Bockrath M. Atomicscale mass sensing using carbon nanotube resonators. Nano Lett 2008; 8:4342-46. https://doi.org/10.1021/nl802181c

[8] Falvo MR, Clary GJ, Taylor RM, Chi V, Brooks JFP, Washburn S, Superfine R. Bending and buckling of carbon nanotubes under large strain. Nature 1997; 389: 582-84. https://doi.org/10.1038/39282
Smirnov VV, Manevitch LI, Strozzi M, Pellicano F. Nonlinear optical vibrations of single-walled carbon nanotubes. 1. Energy exchange and localization of low-frequency oscillations. Physica D 2016; 325 (27): 113-25.

https://doi.org/10.1016/j.physd.2016.03.015

[10] Eringen A. A unified theory of thermo-mechanical materials. Int J Eng Sci 1966; 4(2): 179-02.

https://doi.org/10.1016/0020-7225(66)90022-X

[11] Eringen A, Edelen D. On nonlocal elasticity. Int J Eng Sci 1972; 10: 233-48. https://doi.org/10.1016/0020-7225(72)90039-0

[12] Eringen A. Nonlocal polar elastic continua, Int J Eng Sci 1972; 10(1): 13-16. https://doi.org/10.1016/0020-7225(72)90070-5

[13] Reddy JN. Nonlocal theories for bending, buckling and vibration of beams. Int J Eng Sci 2007; 45: 288-07. https://doi.org/10.1016/j.jjengsci.2007.04.004

[14] Ghayesh MH, Amabili M, Farokhi H. Nonlinear forced vibrations of a microbeam based on the strain gradient elasticity theory. Int J Eng Sci 2013; 63: 52-60.

https://doi.org/10.1016/j.jjengsci.2012.12.001

[15] Lim CW, Islam MZ, Zhang G. A nonlocal finite element method for torsional statics and dynamics of circular nanostructures. Int J Mech Sci 2015; 94: 232-43. https://doi.org/10.1016/j.ijmecsci.2015.03.002

[16] Auffray N, dell'Isola F, Eremeyev VA, Madeo A, Rosi G. Analytical continuum mechanics à la Hamilton-Piola least action principle for second gradient continua and capillary fluids. Math Mech Solids 2015; 20(4): 375-17. https://doi.org/10.1177/1081286513497616

[17] Cazzani A, Stochino F, Turco E. On the whole spectrum of Timoshenko beams. Part I: a theoretical revisitation. Zeitschrift für angewandte Mathematik und Physik 2016; 67(24): 1-30.

https://doi.org/10.1007/s00033-015-0592-0

[18] Li L, Hu Y, Li X. Longitudinal vibration of size-dependent rods via nonlocal strain gradient theory. Int J Eng Sci 2016; 115: 135-44.

https://doi.org/10.1016/j.ijmecsci.2016.06.011

[19] Eltaher MA, Khater ME, Emam Samir A. A review on nonlocal elastic models for bending, buckling, vibrations, and wave propagation of nanoscale beams. Appl Math Modell 2016; 40 (5-6): 4109-28. https://doi.org/10.1016/j.apm.2015.11.026

[20] Bakhshi Khaniki H, Ghayesh MH. A review on the mechanics of carbon nanotube strengthened deformable structures. Eng Struct 2020; 220: 110711. https://doi.org/10.1016/j.engstruct.2020.110711

[21] Ghorbanpour Arani A, Roudbari M.A. Nonlocal piezoelastic surface effect on the vibration of visco-Pasternak coupled boron nitride nanotube system under a moving nanoparticle. Thin Solid Films 2013; 542: 232-41. https://doi.org/10.1016/j.tsf.2013.06.025

[22] Doroudgar Jorshari T, Roudbari MA, Scerrato D, Kouzani A. Vibration suppression of a boron nitride nanotube under a moving nanoparticle using a classical optimal control procedure. Continuum Mech. Thermodyn 2019; 31 (6): 182542.

\section{https://doi.org/10.1007/s00161-019-00813-y}

[23] She GL, Liu HB, Karami B. Resonance analysis of composite curved microbeams reinforced with graphene nanoplatelets. Thin-Walled Struct 2021; 160: 107407. https://doi.org/10.1016/j.tws.2020.107407

[24] Roodgar Saffari P, Fakhraei M, Roudbari MA. Nonlinear vibration of fluid conveying cantilever nanotube resting on visco-Pasternak foundation using nonlocal strain gradient theory. IET Micro Nano Lett IET 2020; 15(3): 183-88. https://doi.org/10.1049/mnl.2019.0420 
[25] Ghorbanpour Arani A, Shajari AR, Amir S, Roudbari MA. Small scale effect on nonlinear vibration of embedded SWBNNTs based on Euler-Bernoulli theory using DQ method. ICNN, Kashan, Iran, 2012.

https://doi.org/10.1016/j.physb.2012.03.065

[26] Bagheri A, Roudbari MA, Mahmoodabadi MJ. Adaptive and sliding mode control for non-linear systems. Int J Adv Manuf Tech 2010; 3(4): 57-62.

[27] Bagheri A, Roudbari MA, Mahmoodabadi MJ. Globally Convergent Adaptive and Sliding Mode Control for Trajectory Tracking with Parameter Uncertainty and Bounded Disturbance. International Symposium on INnovations in Intelligent SysTems and Applications, INISTA 2009, TRABZON, TURKEY.

[28] Roudbari MA. Design of a Robust Adaptive Control (RAC) Of Robotic Manipulators for Trajectory Tracking With Structured and Unstructured Uncertainties. Journal of Communication and Computer 2011; 8: 361-65.

[29] Ghorbanpour Arani A, Roudbari MA, Kiani K. Nonlinear vibration of visco-SWBNNT under moving nanoparticle coupled by Pasternak substrate. Ahrar Science and Technology (In Persian) 2014; 1 (2): 23-30.

[30] Hajjaj AZ, Jaber N, Ilyas S, Alfosail FK, Younis MI. Linear and nonlinear dynamics of micro and nano-resonators: Review of recent advances. Int J Non Linear Mech 2020; 119: 103328.

https://doi.org/10.1016/j.ijnonlinmec.2019.103328

[31] Roodgar Saffari P, Fakhraei M, Roudbari MA. Free vibration and transient response of heterogeneous piezoelectric sandwich annular plate using third-order shear deformation assumption. J Solid Mech 2020; 12 (2): 315-33.

[32] Fakhraei M, Roodgar Saffari P, Roudbari MA. Sizedependent vibration problem of two vertically aligned single walled boron nitride nanotubes conveying fluid in thermal environment via nonlocal strain gradient shell model. J Solid Mech 2021; 13(2): 164-85.

[33] Tubaldi E, Amabili M, Païdoussis MP. Nonlinear dynamics of shells conveying pulsatile flow with pulse-wave propagation. Theory and numerical results for a single harmonic pulsation. J Sound Vib 2017; 396: 217-45.

https://doi.org/10.1016/j.jsv.2017.01.044

[34] Zhou YY, Lü CF, Chen WQ. Bulk wave propagation in layered piezomagnetic/piezoelectric plates with initial stresses or interface imperfections. Compos Struct 2012; 94 (9): $2736-45$.

https://doi.org/10.1016/j.compstruct.2012.04.006

[35] Ghorbanpour Arani A, Roudbari MA, Amir S. Electro-thermo nonlocal wave propagation of the SWBNNTs conveying viscous fluid embedded in a visco elastic medium, ICNN, Kashan, Iran, 2012.

[36] Hosseini SM. Strain gradient and Green-Naghdi-based thermoelastic wave propagation with energy dissipation in a Love-Bishop nanorod resonator under thermal shock loading. Waves Random Complex Media 2021. https://doi.org/10.1080/17455030.2021.1967513

[37] Ghorbanpour Arani A, Roudbari MA. Surface stress, initial stress and Knudsen-dependent flow velocity effects on the electro-thermo nonlocal wave propagation of SWBNNTs. Physica B 2014; 452; 159-65.

https://doi.org/10.1016/j.physb.2014.07.017

[38] Ghorbanpour Arani A, Roudbari MA, Amir S. Longitudinal magnetic field effect on wave propagation of fluid-conveyed SWCNT using Knudsen number and surface considerations. Appl Math Modell 2016; 40 (3): 2025-38. https://doi.org/10.1016/j.apm.2015.09.055

[39] Hosseini SM, Sladek J, Sladek V. Nonlocal coupled photothermoelasticity analysis in a semiconducting micro/nano beam resonator subjected to plasma shock loading: A
Green-Naghdi-based analytical solution. Appl Math Modell 2020; 88: 631-51.

https://doi.org/10.1016/j.apm.2020.06.069

[40] Kiani K. Application of nonlocal higher-order beam theory to transverse wave analysis of magnetically affected forests of single-walled carbon nanotubes. Int J Mech Sci 2018; 138: 116.

https://doi.org/10.1016/j.jimecsci.2018.01.033

[41] Hosseini SM, Zhang C. Nonlocal coupled thermoelastic wave propagation band structures of nano-scale phononic crystal beams based on GN theory with energy dissipation: An analytical solution. Wave Motion 2020; 92: 102429. https://doi.org/10.1016/j.wavemoti.2019.102429

[42] Shahsavari H, Talebitooti R, Kornokar M. Analysis of wave propagation through functionally graded porous cylindrical structures considering the transfer matrix method. ThinWalled Struct 2021; 162: 107212. https://doi.org/10.1016/j.tws.2020.107212

[43] Karimipour I, Tadi Beni Y, Arvin H, Akbarzadeh AH. Dynamic wave propagation in micro-torus structures: Implementing a 3D physically realistic theory. Thin-Walled Struct 2021; 165 107995.

https://doi.org/10.1016/j.tws.2021.107995

[44] Yuan Y, Zhao X, Zhao Y., Sahmani S, Safaei B. Dynamic stability of nonlocal strain gradient FGM truncated conical microshells integrated with magnetostrictive facesheets resting on a nonlinear viscoelastic foundation. Thin-Walled Struct 2021; 159: 107249 https://doi.org/10.1016/j.tws.2020.107249

[45] Zhang $\mathrm{H}$, Challamel N, Wang CM, Zhang YP. Buckling of multiply connected bar-chain and its associated continualized nonlocal model. Int J Mech Sci 2019; 150: 168-75. https://doi.org/10.1016/j.jmecsci.2018.10.015

[46] Chen D, Yang J, Kitipornchai S. Elastic buckling and static bending of shear deformable functionally graded porous beam. Compos Struct 2015; 133: 54-61. https://doi.org/10.1016/j.compstruct.2015.07.052

[47] Yang J, Wu H, Kitipornchai S. Buckling and postbuckling of functionally graded multilayer graphene platelet-reinforced composite beams. Compos Struct 2017; 161: 111-18. https://doi.org/10.1016/j.compstruct.2016.11.048

[48] Kitipornchai S, Chen D, Yang J. Free vibration and elastic buckling of functionally graded porous beams reinforced by graphene platelets. Materials \& Design 2017; 116: 656-65. https://doi.org/10.1016/j.matdes.2016.12.061

[49] Liu D, Kitipornchai S, Chen WQ, Yang J. Three-dimensional buckling and free vibration analyses of initially stressed functionally graded graphene reinforced composite cylindrical shell. Compos Struct 2018; 189: 560-69. https://doi.org/10.1016/j.compstruct.2018.01.106

[50] Chen D, Yang J, Kitipornchai S. Buckling and bending analyses of a novel functionally graded porous plate using Chebyshev-Ritz method. Arch Civ Mech Eng 2019; 19 (1): 157-70.

\section{https://doi.org/10.1016/j.acme.2018.09.004}

[51] Gao K, Huang Q, Kitipornchai S, Yang J. Nonlinear dynamic buckling of functionally graded porous beams. Mech Adv Mater Struct 2019; 1-12.

[52] Dastjerdi S, Akgöz B, Civalek Ö. On the effect of viscoelasticity on behavior of gyroscopes. Int J Eng Sci 2020; 149: 103236 https://doi.org/10.1016/j.ijengsci.2020.103236

[53] Amabili M, Breslavsky ID, Reddy JN. Nonlinear higher-order shell theory for incompressible biological hyperelastic materials. Comput Method Appl M 2019; 346: 841-861. https://doi.org/10.1016/j.cma.2018.09.023

[54] Jung WY, Park WT, Han SC. Bending and vibration analysis of S-FGM microplates embedded in Pasternak elastic 
medium using the modified couple stress theory. Int $\mathrm{J}$ Mech Sci 2014; 87: 150-62.

https://doi.org/10.1016/j.ijmecsci.2014.05.025

[55] Koutsoumaris CC, Eptaimeros KG. Eringen's Nonlocal Integral Elasticity and Applications for Structural Models. Size-Dependent Continuum Mechanics Approaches 2021; 51-93.

https://doi.org/10.1007/978-3-030-63050-8 2

[56] Lazar M, Maugin GA, Aifantis EC. On a theory of nonlocal elasticity of bi-Helmholtz type and some applications. Int $J$ Solids Struct 2006; 43(6): 1404-21. https://doi.org/10.1016/j.ijsolstr.2005.04.027

[57] Russo R, Girot Mata FA, Forest S, Jacquin D. A review on strain gradient plasticity approaches in simulation of manufacturing processes. J Manuf Mater Process 2020; 4(87): 1-29.

https://doi.org/10.3390/jmmp4030087

[58] Wang XQ, Lee JD. Micromorphic theory: a gateway to nano worl. Int J Smart Nano Mater 2010; 1(2): 115-35. https://doi.org/10.1080/19475411.2010.484207

[59] Vernerey F, Liu WK, Moran B. Multi-scale micromorphic theory for hierarchical materials. J Mech Phys Solids 2007; 55: 2603-51.

https://doi.org/10.1016/j.jmps.2007.04.008

[60] Chen Y, Lee J, Xiong L. A generalized continuum theory and its relation to micromorphic theory. J Eng Mech 2009; 14955.

https://doi.org/10.1061/(ASCE)0733-9399(2009)135:3(149)

[61] Barati MR. A general nonlocal stress-strain gradient theory for forced vibration analysis of heterogeneous porous nanoplates. Eur J Mech A Solids 2018; 67: 15-30. https://doi.org/10.1016/j.euromechsol.2017.09.001

[62] Ebrahimi F, Barati MR, Dabbagh A. A nonlocal strain gradient theory for wave propagation analysis in temperature-dependent inhomogeneous nanoplates. Int J Eng Sci 2016; 107: 169-82.

https://doi.org/10.1016/j.ijengsci.2016.07.008

[63] Khaniki HB, Ghayesh MH, Amabili M. A review on the statics and dynamics of electrically actuated nano and micro structures. Int J Non Linear Mech 2021; 129: 103658. https://doi.org/10.1016/j.ijnonlinmec.2020.103658

[64] Roudbari MA, Doroudgar Jorshari T, Lu C, Ansari R, Kouzani A, Amabili M. A review of size-dependent continuum mechanics models for micro-and nano-structures. ThinWalled Struct 2022; 170: 108562.

https://doi.org/10.1016/j.tws.2021.108562

[65] Awrejcewicz J, Krysko AV, Zhigalov MV, Krysko VA. Sizedependent theories of beams, plates and shells, Mathematical Modelling and Numerical Analysis of SizeDependent Structural Members in Temperature Fields. Springer Cham 2021; 25-78. https://doi.org/10.1007/978-3-030-55993-9 2

[66] Thai HT, Vo TP, Nguyen TK, Kim SE. A review of continuum mechanics models for size-dependent analysis of beams and plates. Compos Struct 2017; 177(1): 196-19. https://doi.org/10.1016/j.compstruct.2017.06.040

[67] Hosseini M, Hadi A, Malekshahi A, shishesaz M. A review of size-dependent elasticity for nanostructures. J Appl Comput 2018; 49: 197-11.

[68] lijima S, Brabec C, Maiti A, Bernholc J. Structural flexibility of carbon nanotubes. J Chem Phys 1996; 104: 2089-92. https://doi.org/10.1063/1.470966

[69] Lim CW, Zhang G, Reddy JN. A higher-order nonlocal elasticity and strain gradient theory and its applications in wave propagation. J Mech Phys Solids 2015; 78: 298-13. https://doi.org/10.1016/j.jmps.2015.02.001
[70] Ghorbanpour Arani A, Roudbari MA, Amir S. Nonlocal vibration of SWBNNT embedded in bundle of CNTs under a moving nanoparticle. Physica B 2012; 407 (17): 3646-53. https://doi.org/10.1016/j.physb.2012.05.043

[71] Ghorbanpour Arani A, Roudbari MA, Kiani K. Vibration of double-walled carbon nanotubes coupled by temperaturedependent medium under a moving nanoparticle with multi physical fields. Mech Adv Mater Struct 2016; 23 (3): 281-91. https://doi.org/10.1080/15376494.2014.952853

[72] Ghorbanpour Arani A, Jalilvand A, Ghaffari M, Taleb Mazraehshahi M, Kolahchi R, Roudbari MA, Amir S. Nonlinear pull-in instability of boron nitride nano-switches considering electrostatic and Casimir forces. Sci Iran 2014; 21 (3): 1183-96.

[73] Ghorbanpour Arani A, Karamali Ravandi A, Roudbari MA, Azizkhani MB, Hafizi A. Axial and transverse vibration of SWBNNT system coupled Pasternak foundation under a moving nanoparticle using Timoshenko beam theory. J Solid Mech 2015; 7 (3): 239-54.

[74] Ghorbanpour Arani A, Hafizi Bidgoli A, Karamali Ravandi A. Roudbari MA, Amir S, Azizkhani MB. Induced nonlocal electric wave propagation of boron nitride nanotubes. J Mech Sci Technol 2013; 27 (10): 3063-71. https://doi.org/10.1007/s12206-013-0705-7

[75] Roudbari MA, Doroudgar Jorshari T, Ghorbanpour Arani A Lü C, Rabczuk T. Transient responses of two mutually interacting single-walled boron nitride nanotubes induced by a moving nanoparticle. Eur J Mech A Solids 2020; 82: 103978.

https://doi.org/10.1016/j.euromechsol.2020.103978

[76] Lu L, Guo X, Zhao J. A unified nonlocal strain gradient model for nanobeams and the importance of higher order terms. Int J Eng Sci 2017; 119: 265-77. https://doi.org/10.1016/j.ijengsci.2017.06.024

[77] Arefi M, Kiani M, Rabczuk T. Application of nonlocal strain gradient theory to size dependent bending analysis of a sandwich porous nanoplate integrated with piezomagnetic face-sheets. Compos Part B-Eng 2019; 168: 320-33. https://doi.org/10.1016/j.compositesb.2019.02.057

[78] Zaera R, Serrano Ó, Fernández-Sáez J. Non-standard and constitutive boundary conditions in nonlocal strain gradient elasticity. Meccanica 2020; 55: 469-79. https://doi.org/10.1007/s11012-019-01122-z

[79] Karami M, Janghorban M. On the mechanics of functionally graded nanoshells. Int J Eng Sci 2020; 153: 103309. https://doi.org/10.1016/j.ijengsci.2020.103309

[80] Roudbari MA, Ansari R. Single-walled boron nitride nanotube as nano-sensor. Continuum Mech Thermodyn 2020; 32: 729748.

https://doi.org/10.1007/s00161-018-0719-6

[81] Allam MNM, Radwan AF. Nonlocal strain gradient theory for bending, buckling, and vibration of viscoelastic functionally graded curved nanobeam embedded in an elastic medium. Adv Mech Eng 2019; 11(4): 1-15.

https://doi.org/10.1177/1687814019837067

[82] Xu XJ, Wang XC, Zheng ML, Ma Z. Bending and buckling of nonlocal strain gradient elastic beams. Compos Struct 2017; 160: 366-77.

https://doi.org/10.1016/j.compstruct.2016.10.038

[83] Barretta R, Candadija M, Marotti De Sciarra F. Modified nonlocal strain gradient elasticity for nano-rods and application to carbon nanotubes. Appl Sci 2019; 9 (514): 121.

https://doi.org/10.3390/app9030514

[84] Gao Y, Xiao WS, Zhu H. Nonlinear bending of functionally graded porous nanobeam subjected to multiple physical load based on nonlocal strain gradient theory. Steel Compos Struct 2019; 31: 469-88. 
[85] Bedia WA, Houari MSA, Bessaim A, Bousahla AA, Tounsi A, Saeed T, Alhodaly MS. A New Hyperbolic Two-Unknown Beam Model for Bending and Buckling Analysis of a Nonlocal Strain Gradient Nanobeams. Nano Res 2019; 57: 175-91. https://doi.org/10.4028/www.scientific.net/JNanoR.57.175

[86] Farajpour A, Haeri Yazdi MR, Rastgoo A, Mohammadi M. A higher-order nonlocal strain gradient plate model for buckling of orthotropic nanoplates in thermal environment. Acta Mech 2016; 227: 1849-67. https://doi.org/10.1007/s00707-016-1605-6

[87] Li X, Li L, Hu Y, Ding Z, Deng W. Bending, buckling and vibration of axially functionally graded beams based on nonlocal strain gradient theory. Compos Struct 2017; 165: $250-65$ https://doi.org/10.1016/j.compstruct.2017.01.032

[88] Zhong B, Shen H, Liu J, Wang Y, Zhang Y. Deep postbuckling and nonlinear bending behaviors of nanobeams with nonlocal and strain gradient effects. Appl Math Mech 2019; 40: 515-48. https://doi.org/10.1007/s10483-019-2482-9

[89] Li L, Hu Y. Post-buckling analysis of functionally graded nanobeams incorporating nonlocal stress and microstructuredependent strain gradient effects. Int. J. Mech. Sci. 2017; 120: $159-70$. https://doi.org/10.1016/j.ijmecsci.2016.11.025

[90] Mao JJ, Zhang W. Buckling and post-buckling analyses of functionally graded graphene reinforced piezoelectric plate subjected to electric potential and axial forces. Compos Struct 2019; 216: 392-05. https://doi.org/10.1016/j.compstruct.2019.02.095

[91] Sahmani S, Safaei B. Nonlinear free vibrations of bidirectional functionally graded micro/nano-beams including nonlocal stress and microstructural strain gradient size effects. Thin-Walled Struct 2019; 140: 342-56. https://doi.org/10.1016/j.tws.2019.03.045

[92] Ghayesh MH, Farokhi H, Farajpour A. Global dynamics of fluid conveying nanotubes. Int J Eng Sci 2019; 135: 37-57. https://doi.org/10.1016/j.ijengsci.2018.11.003
[93] Roudbari MA, Doroudgar Jorshari T. Vibrational control scrutiny of physically affected SWCNT acted upon by a moving nanoparticle in the framework of nonlocal-strain gradient theory. J Braz Soc Mech Sci Eng 2018; 40: 499. https://doi.org/10.1007/s40430-018-1421-0

[94] Wu Q, Chen $H$, Gao W. Nonlocal strain gradient forced vibrations of FG-GPLRC nanocomposite microbeams. Eng Comput 2020; 36: 1739-50. https://doi.org/10.1007/s00366-019-00794-1

[95] Mahmoudpour E. Nonlinear resonant behavior of thick multilayered nanoplates via nonlocal strain gradient elasticity theory. Acta Mech 2020; 231: 2651-67. https://doi.org/10.1007/s00707-020-02636-4

[96] Vahidi-Moghaddam AM, Rajaei A., Azadi Yazdi E, Eghtesad $M$, Necsulescu DS. Nonlinear forced vibrations of nonlocal strain gradient microbeams. Mech Based Des Struct Mach 2020. https://doi.org/10.1080/15397734.2020.1860773

[97] Tang Y, Liu Y, Zhao D. Viscoelastic wave propagation in the viscoelastic single walled carbon nanotubes based on nonlocal strain gradient theory. Physica E 2016; 84: 202-208. https://doi.org/10.1016/j.physe.2016.06.007

[98] Norouzzadeh A, Ansari R, Rouhi H. An analytical study on wave propagation in functionally graded nano-beams/tubes based on the integral formulation of nonlocal elasticity. Waves Random Complex Medium 2019; 1-19. https://doi.org/10.1080/17455030.2018.1543979

[99] Huang $\mathrm{Y}$, Wei $\mathrm{P}, \mathrm{Xu} \mathrm{Y}, \mathrm{Li} \mathrm{Y}$. Modelling flexural wave propagation by the nonlocal strain gradient elasticity with fractional derivatives. Math Mech Solids 2021. https://doi.org/10.1177/1081286521991206

[100] Huang Y, Wei P. Modelling the flexural waves in a nanoplate based on the fractional order nonlocal strain gradient elasticity and thermoelasticity. Compos Struct 2021; 266: 113793

https://doi.org/10.1016/j.compstruct.2021.113793 